\title{
INTERACCIÓN INSTITUCIONAL Y SOCIALIZACIÓN PROFESIONAL: REPRESENTACIONES SOBRE EL TRATAMIENTO PSIQUIÁTRICO EN ENTREVISTAS DE ADMISIÓN EN SALUD MENTAL
}

\author{
JUAN EDUARDO BONNIN \\ CONICET
}

\section{RESUMEN}

El objetivo de este trabajo es observar el impacto de la socialización profesional en la interacción entre psicólogos y pacientes en los consultorios externos de salud mental de un hospital público de la ciudad de Buenos Aires, Argentina. Desde una perspectiva crítica que combina el análisis de la conversación con el análisis del discurso, analizamos entrevistas de admisión realizadas por profesionales de planta del hospital y por profesionales precarizados con menor experiencia clínica. Esto nos permite observar representaciones distintas acerca del tratamiento psiquiátrico, por un lado, y de la relación del paciente con la medicación, por otro. El análisis realizado permite afirmar que las condiciones de trabajo precario en salud mental impactan en el ingreso de los pacientes al sistema y en las percepciones que éstos puedan desarrollar de la salud mental en general y el tratamiento psiquiátrico en particular.

PALABRAS CLAVE: Comunicación médico-paciente, salud mental, socialización profesional, condiciones de trabajo, psiquiatría, psicología.

\section{ABSTRACT}

The main purpose of this article is to observe the effects of professional socialization in the interaction between psychologists and patients at an outpatient service of mental health at a public hospital in Buenos Aires, Argentina. Assuming a critical perspective that combines discourse analysis and conversation analysis, we analyze admission interviews conducted by employees of the hospital and by precarized workers with less clinical experience. We observe different representations about psychiatric treatment, on one hand, and about the relationship of the patient with medication, on the other. Analysis allows us to 
state that precarious labor conditions in mental healthcare affect the admission of patients to the system and the perceptions they may develop about mental healthcare in general, and about psychiatric treatment in particular.

KEYWORDS: Doctor-patient communication, mental healthcare, professional socialization, working conditions, Psychiatry, Psychology.

\section{INTRODUCCIÓN: PRECARIEDAD LABORAL Y REPRESENTACIONES DISCURSIVAS}

Las investigaciones sobre la interacción en entornos médicos se integran habitualmente en algunas pocas grandes corrientes. La perspectiva más tradicional, fundada a mediados de los años setenta, es la del análisis de la conversación "médico-paciente", una mirada detallada de los recursos semióticos (fundamentalmente verbales) desplegados en la relación diádica entre un profesional de la salud y un lego (cfr. Heritage y Maynard, 2010; Peräkylä et al., 2008). Más recientemente, a raíz de trabajos como los de Sarangi y Roberts (1998), Candlin y Candlin (2003) y los reunidos por Iedema (2007), la perspectiva excesivamente minimalista del análisis de la conversación se ha ampliado a lo que denominan "interacción en entornos médicos" (medical settings), dentro del marco más general de la comunicación institucional.

Uno de los puntos poco discutidos en estos trabajos es el referido a la socialización profesional y las condiciones de trabajo de los profesionales involucrados en la interacción. La organización de los roles en la díada "médico-paciente" proyecta una imagen de homogeneidad en los sujetos que los encarnan que dista de corresponderse con profesionales diversos en términos ideológicos, sociales, étnicos, de género, e incluso laborales. En nuestra investigación, estos aspectos se han revelado centrales para comprender algunas características de la interacción efectivamente desarrollada entre profesionales y pacientes.

A lo largo de cuatro años de compromiso de campo en los consultorios externos de salud mental de un hospital público de la Ciudad de Buenos Aires, hemos observado que el rol del "profesional" de la salud mental (vgr. psicólogos y, en menor medida, psiquiatras) no es construido del mismo modo por todos los sujetos, ni todos se comportan del mismo modo durante la interacción clínica. Una distinción fundamental es entre personal asalariado ("de planta" o "residentes") y no asalariado ("concurrentes" y "cursistas"): puesto que estos últimos no disponen de la misma cantidad ni calidad de tiempo para la socialización profesional que sus colegas, su contacto con profesionales médicos de la salud mental (psiquiatras) es menor $y$, en consecuencia, sus representaciones acerca del tratamiento psiquiátri- 
co y la medicación son diferentes. Esta diferencia, como veremos, puede proyectarse sobre los pacientes y su relación con el tratamiento.

En lo que sigue presentaremos, en primer lugar, la perspectiva de análisis que proponemos acerca de la comunicación entre profesionales y pacientes en entornos médicos. En segundo lugar, describiremos las condiciones laborales y sus efectos en la socialización profesional de los actores del sistema público de salud mental de la Ciudad de Buenos Aires. Con estos datos, analizaremos pasajes de interacción entre psicólogos "de planta" y precarizados con pacientes, para observar sus diferentes representaciones acerca del tratamiento psiquiátrico, primero, y acerca de la relación del paciente con la medicación psiquiátrica, después. Finalmente, propondremos una discusión evaluativa de los resultados alcanzados para vincular el fenómeno observado con las políticas de salud mental.

\section{SOCIALIZACIÓN PROFESIONAL, CONDICIONES DE TRABAJO Y COMUNICACIÓN MÉDICA}

La interacción verbal entre profesionales y pacientes en entornos médicos es habitualmente caracterizada desde una mirada estructural acerca de los sistemas sociales y la distribución de roles: el rol del enfermo y el rol del médico (Parsons, 1975). Comprendiendo que esta caracterización corresponde a los "principios generales" (Heritage y Clayman, 2010: 119) del contexto médico, las aproximaciones etnográficas y empíricas a los procesos sociales en juego en dichas situaciones son escasas o prácticamente inexistentes. Volúmenes actuales de referencia sobre la interacción entre médicos y pacientes (Heritage y Maynard, 2006; Heritage y Clayman, 2010: 119 y ss.) adoptan la descripción parsoniana de los roles sin cuestionar el salto cualitativo entre estructura social y datos situacionales. Como señala Blommaert (2005: 51-52), de la combinación de supuestos teóricos definidos a priori y etnografías superficiales surge, con frecuencia, una contextualización poco crítica que sitúa a la conversación en un marco esquemático y estático de "situación no discursiva". Este tipo de estudios deja de lado la negociación cotidiana de los roles, los procesos históricos y las tradiciones discursivas presentes en cada institución, la diversidad ideológica, social, laboral, étnica y de género de los participantes, etc. (Fochsen, Deshpande y Thorson, 2006; Fox et al., 2009). Las secciones destinadas a la descripción contextual de los datos (data and settings) a menudo se contentan con señalar rápidamente la especialidad médica y luego se detienen en aspectos metodológicos como la cantidad de entrevistas, su duración, etc. Aspectos clave como el carácter público/privado de la institución, la expe- 
riencia laboral del médico, la experiencia previa del paciente, etc., son generalmente obviados (Bonnin, 2013) ${ }^{1}$.

Desde una perspectiva discursiva, estudios sobre la comunicación organizacional en salud (Iedema, 2005a, b, 2007; Sarangi, 2004, 2011; Candlin y Candlin, 2003; Crawford, Brown y Mullany, 2007) muestran la necesidad de una aproximación etnográfica que supere la díada médico-paciente en el consultorio. De este modo, adoptar al hospital como contexto permite "to inform policy makers, health managers and hospital clinicians about how work is changing in these sites and what these changes entrain for clinical professionals in interactive-discursive terms" (Iedema, 2005a: 244). Adoptando este punto de vista, la negociación y reconfiguración de los roles y situaciones institucionales no es excepcional sino constitutiva del entorno médico (Cicourel, 1999; Sarangi, 2011; Bonnin, 2013), incluyendo parámetros contextuales sujetos a la variación basada, por ejemplo, en la experiencia previa de los participantes (Candlin y Candlin, 2007).

Una de las maneras de vincular estos procesos locales de interacción con procesos sociales más amplios es a través de las trayectorias de los participantes y su inserción estructural en el evento. El concepto de "socialización" nos permite realizar esa vinculación puesto que no opone un "adentro" y un "afuera" de la situación ni compara distintos niveles (macro/micro, institucional/situacional, etc.). Por el contrario, son los mismos sujetos participantes quienes, a través de sus trayectorias profesionales, establecen un continuum entre los diversos procesos sociales involucrados en el contexto hospitalario.

La socialización puede definirse como un proceso de desarrollo subjetivo que se produce por la interacción constante entre el individuo y el entorno (Conway, 1983). La literatura especializada suele diferenciar entre "socialización primaria" (los vínculos fuertes desarrollados durante la niñez) y "secundaria" (espacios de socialización más amplios en la integración del individuo a la sociedad). La socialización no es un simple aprendizaje pasivo de roles sociales apropiados, sino un proceso activo por el cual el mundo objetivo de la realidad es internalizado y se vuelve subjetivamen-

${ }^{1}$ Esta debilidad ha sido señalada por Cordella (2004) en una amplia variedad de trabajos sobre comunicación médico-paciente. En el campo de la sociología de la salud, May (2007) ha señalado una situación análoga, proponiendo en cambio una situación dinámica y multidimensional en la que el Estado y actores corporativos participan del encuentro clínico como parte de un complejo conjunto de recursos y prácticas organizacionales, institucionales y disciplinarias. Notables excepciones las constituyen los trabajos de Moyer (2011) y Moyer y Codó (2002), que realizan un detallado análisis etnográfico y sociológico de la interacción en un hospital público en Barcelona, España. De manera semejante, los trabajos de Pardo y Lerner (2001) y Pardo y Buscaglia (2008) contextualizan críticamente la relación entre discurso y salud mental, aunque no en entornos hospitalarios. 
te significativo (Clouder, 2003: 215) en el contraste entre el mundo de los fenómenos socialmente construidos y las expectativas del individuo. En el campo profesional, específicamente, la socialización puede conceptualizarse como un proceso de desarrollo de "a unique voice, perspective, or personal and professional world-view" (Clark, 1997: 442). Como señala Clark (1997), la socialización profesional, tal como se desarrolla en el lugar de trabajo, incluye la adquisición de saberes y valores asociados a la práctica y los participantes de las actividades profesionales.

Para el caso de los psicólogos que trabajan en el sistema público de salud mental en la Ciudad de Buenos Aires, la socialización profesional en el entorno hospitalario impacta, entre otros aspectos, en las representaciones que tienen acerca de la medicina y lo que denominan el "discurso médico" en general. Como se ha señalado (Lakoff, 2006), existe una importante resistencia por parte de los jóvenes graduados en psicología con respecto al saber médico, que consideran muchas veces un intento ilegítimo de "biologización de la psique" (Bonnin, 2014c). El proceso de socialización profesional, sin embargo, permite la comunicación interprofesional -a través de la negociación de saberes, significados y valores- con sus colegas médicos psiquiatras. Esta negociación, sin embargo, depende de las condiciones laborales y sociales más amplias en las que se lleva a cabo el proceso de socialización.

En efecto, la demanda de salud mental en los hospitales públicos se ha quintuplicado en pocos años: de acuerdo a datos del Hospital en el que realizamos trabajo de campo, de las 493 atenciones realizadas en 2007 por los consultorios de salud mental se pasó a 2864 en 2011. ¿Cómo hacen frente los servicios de salud mental a este aumento en la cantidad de pacientes si no aumenta la cantidad de profesionales?

Frente a la carencia de recursos por parte del Estado, son los equipos de salud mental en el terreno quienes se hacen cargo del diseño y la implementación de estrategias de inclusión en salud. Con los recursos (humanos) disponibles, despliegan tácticas cotidianas destinadas a suplir la falta de políticas estatales inclusivas. En tal sentido, frente a la escasez de psicólogos rentados ("de planta" y "residentes") recurren a jóvenes profesionales en formación, sin salario, que se enmarcan en las figuras del "concurrente" y del "cursista", las dos formas más visibles de precarización en el sector salud (Novick y Galin, 2003).

La figura de mayor estabilidad laboral es la del profesional "de planta", que tiene una relación salarial formal con el Hospital público, se encuentra amparado bajo un convenio colectivo de trabajo y es sujeto pleno de derechos y deberes laborales. Se trata de cargos a los que se accede por concurso público de antecedentes, entre los cuales tiene mucho peso la previa realización de una residencia. 
Las "residencias" son prácticas de capacitación de posgrado para profesionales que se insertan, de este modo, en el sistema de salud de la Ciudad de Buenos Aires. Al ser rentadas, exigen de los profesionales una dedicación exclusiva a tiempo completo, desarrollando "actividades asistenciales programadas y supervisadas en instituciones dependientes del Sistema de Salud del Gobierno de la Ciudad de Buenos Aires" durante un período de cuatro años ${ }^{2}$. Cada año se convoca a un examen, de cuyo orden de mérito se seleccionará a los primeros puestos como "residentes". Aunque no hay cifras públicas con la cantidad de personas que ingresan como residentes, Antman (2007) señala que sólo el 3\% de los postulantes a la residencia en "psicología clínica" (recientemente renombrada "salud mental") obtienen un cargo rentado. En cifras absolutas, si tenemos en cuenta que en el año 2015 se presentaron 477 postulantes, deberíamos concluir que ingresaron alrededor de 14 personas destinadas a alguno de los 13 hospitales generales de agudos o los 19 hospitales especializados ${ }^{3}$.

De entre los profesionales que rinden el examen de residencia, el 3\% superior accede a la misma; el siguiente $28 \%$ es seleccionado en la categoría de "concurrente" (Antman, 2003). Las concurrencias "corresponden a un sistema honorario (no rentado) de capacitación profesional de posgrado, a tiempo parcial desarrollado bajo condiciones de programación y supervisión" ${ }^{4}$. Con una duración de cinco años, estos profesionales trabajan gratuitamente en el sistema de salud y, a cambio, reciben cursos de posgrado sin titulación. Según datos presentados por Antman (2007), los concurrentes representan el $58 \%$ del total de psicólogos trabajando en la "Red de Servicios de Salud Mental" de la Ciudad de Buenos Aires. Esto significa que seis de cada diez psicólogos que atienden pacientes en los hospitales públicos no viven de ese trabajo, sino que lo realizan en el tiempo que su trabajo remunerado se lo permite, con la dedicación y el tiempo que su voluntad y sus condiciones hacen posible.

Por último, en el hospital en el que desarrollamos nuestro trabajo de campo existe la figura de los "cursistas", profesionales jóvenes que no son residentes ni concurrentes (algunos fallaron en sus exámenes de residencia, otros no los rindieron), que atienden pacientes en el marco de

${ }^{2}<$ http://www.buenosaires.gob.ar/salud/residenciasyconcurrencias/presentacion-deresidencia-y-concurrencias $>$.

${ }^{3}$ Los datos de Antman (2003, 2007) fueron recogidos en el año 2003. Careciendo de cifras oficiales, es la única fuente a la que podemos recurrir, aunque es preciso señalar el carácter aproximado de estos datos con respecto al momento de nuestro trabajo de campo (entre 2012 y 2014).

${ }^{4}<$ http://www.buenosaires.gob.ar/salud/residenciasyconcurrencias/presentacion-deresidencia-y-concurrencias $>$. 
equipos de diversas especialidades y, a cambio, reciben supervisión y son admitidos gratuitamente a los cursos que los jefes de equipo dictan en el servicio.

Residentes y cursistas son la categoría más numerosa de psicólogos que trabajan en el sistema de salud mental de la ciudad de Buenos Aires. Este trabajo se ve lógicamente resentido: por una parte, como el sistema de concurrencia tiene un objetivo de formación en el lugar de trabajo, carecen de experiencia clínica previa. A diferencia de los residentes, sin embargo, no se dedican a tiempo completo ni reciben una remuneración por su trabajo; en consecuencia, no pueden adquirir la experiencia en el mismo tiempo ni pueden destinarle la misma cantidad de horas, puesto que su trabajo asalariado es otro. A semejanza de ellos, sin embargo, tratan pacientes y realizan un conjunto de tareas sin las cuales los servicios de salud mental no podrían funcionar.

Estos diversos procesos de socialización profesional en el espacio hospitalario impactan en su desempeño interaccional con los pacientes. Nosotros nos detendremos en uno de ellos: al carecer de contacto frecuente, y de formación específica, con profesionales médicos psiquiatras, las representaciones acerca del tratamiento psiquiátrico y el uso de la medicación son diferentes a los del caso de los psicólogos de planta. Esta situación, obvia al tratarse de jóvenes en formación, no debería ser problemática en la medida en que trabajarán en pares con profesionales ya formados. Sin embargo, al aumentar la demanda y no la planta de profesionales, quedan a cargo de la atención de los pacientes: con una insuficiente socialización profesional, desarrollada en condiciones precarias de trabajo, los saberes, representaciones y valores asociados a la psiquiatría impactan en la interacción con los pacientes, sus representaciones sobre el tratamiento psiquiátrico y el uso de la medicación ${ }^{5}$.

\section{Datos, contexto y metodología}

Las instituciones de salud pública en Argentina, al menos en la Ciudad de Buenos Aires, muestran notables diferencias con las caracterizaciones disponibles para otros países. Mientras que Wodak (2006: 682) señala que,

${ }^{5}$ NB: la terminología empleada tanto por el Gobierno de la Ciudad de Buenos Aires como por algunas investigaciones sobre el tema describe el trabajo de los concurrentes como "ad honorem". En nuestro caso, puesto que cuestionamos la naturaleza precaria de este trabajo, no utilizamos esta designación, en la medida en la cual consideramos que es una forma de explotación de profesionales jóvenes para sostener el déficit presupuestario del sistema de salud pública, el cual se aprovecha de -y no suma a- su honor. 
en los hospitales, "contradiction and conflicts are often concealed and not discussed or exposed openly", los conflictos laborales y gremiales forman parte del ambiente hospitalario porteño, y los folletos, afiches y asambleas de trabajadores forman parte de la vida cotidiana. La asimetría estructural de roles y la "orientación al estatus" de la interacción son constantemente cuestionados por los pacientes; testimonios recientes de este cuestionamiento son los recurrentes episodios de violencia física hacia el personal médico ocurridos en los últimos años (cfr. Organización Panamericana de la Salud, 2012).

En el marco de nuestra investigación, hemos realizado cuatro años de etnografía en el "equipo de admisión" de los consultorios externos de salud mental de un hospital público de la Ciudad de Buenos Aires entre 2011 y 2014. En ese marco, hemos participado de las diversas actividades del equipo en los consultorios externos de salud mental, incluyendo la sala de espera, actividades de capacitación, jornadas y las entrevistas de admisión, o "primeras consultas", propiamente dichas. Observamos y/o audio-grabamos 108 entrevistas, con una duración aproximada de veinte minutos cada una, en todos los casos con el consentimiento informado de los participantes. Por motivos de confidencialidad, no identificamos el hospital ni a los participantes; en cambio, reemplazamos todos los nombres propios por letras seleccionadas al azar.

El equipo de admisión fue constituido por el Jefe de servicio de los consultorios externos para concentrar la demanda de tratamiento en un actor institucional compuesto por representantes de todas las especialidades: terapia de adultos, familiar, de adicciones, etc. Puesto que se trata de un equipo ad hoc, constituido para hacer frente a un problema práctico, pero sin reconocimiento institucional ni salarial, los representantes de los diversos equipos participan movidos por la voluntad, de manera que en su enorme mayoría se trata de personal precarizado. Con una composición cambiante, puesto que sus miembros condicionan la participación a sus posibilidades laborales, sus doce miembros más estables se dividen en: dos psiquiatras de planta, tres psicólogos de planta y siete profesionales sin salario, entre concurrentes y cursistas.

El equipo de admisión realiza las entrevistas durante una mañana, un día fijo por semana, en parejas que típicamente se componen por un personal rentado y más experimentado, que oficia como "psicólogo a cargo", y uno sin salario, que hemos caracterizado como "psicólogo asistente" (sobre los roles, cfr. Bonnin, 2013). Habitualmente se realizan entre quince y veinte entrevistas, a razón de dos o tres entrevistas por pareja. Aunque se busca que haya siempre un profesional de planta a cargo, es frecuente que al menos una pareja esté integrada por dos profesionales precarizados.

Las entrevistas que estamos analizando tienen una estructura tripartita, definida de modo informal pero recurrente: 
1) La primera parte consiste en el llenado de un formulario estadísticoepidemiológico provisto por el Ministerio de Salud de la Ciudad de Buenos Aires. Aunque los pacientes suelen desplegar estrategias expansivas durante esta movida (por ejemplo, "contestar más allá de lo pedido", "answering more than the question", Stivers y Heritage, 2001; Bonnin, 2014b), los profesionales de mayor experiencia suelen descartar las secuencias expansivas y enfocarse en la tarea entre manos. Aquellos que tienen menos experiencia, en cambio, pueden abandonar la actividad que están desarrollando y adoptar la agenda del paciente (Bonnin, 2014b), moviéndose a la segunda parte de la entrevista.

2) La segunda fase es designada por los profesionales del servicio como "motivo de consulta". Se trata de una actividad que involucra secuencias de distinto tipo, las cuales por momentos pueden evocar la fase de "history taking" típica de las entrevistas médicas (por ejemplo la secuencia Pregunta - Respuesta - Evaluación; cfr. infra ex. 2, líneas 13-15; cfr. Heritage y Clayman, 2010) pero que delegan buena parte de la responsabilidad por el avance de la interacción en el destinatario, es decir, el paciente (Peräkylä, 2008). En tal sentido, expresiones continuativas como "ajá", o "hmm" son típicas de la interacción psicoanalítica y emergen recurrentemente al conversar sobre el "motivo de consulta".

3) Una vez recabada suficiente información para elaborar un diagnóstico provisorio y una recomendación de tratamiento (Bonnin, 2014c), el analista comienza una nueva etapa más burocrática o administrativa, en la que informa las posibilidades de tratamiento, rechaza o acepta al paciente (según criterios de diversa índole; cfr. Bonnin, 2014b) y, en caso de aceptarlo, solicita información de contacto y da algunas recomendaciones generales hasta el momento de comenzar el tratamiento propiamente dicho.

\section{ANÁLISIS}

Puesto que nos interesa observar las representaciones de psicólogos con distinta inserción laboral (y, en consecuencia, socialización profesional) acerca del tratamiento psiquiátrico y la medicación, tomamos aquellas secuencias en las cuales se discuten ambos temas.

Los datos son analizados desde una perspectiva discursiva que combina el interés ideológico y político del análisis del discurso (Arnoux, 2006) con un examen detallado de la interacción (Bolívar, 2010), especialmente en lo referido a la organización secuencial de la conversación. Desde esta perspectiva, conceptos descriptivos provistos por el análisis de la conver- 
sación (como secuencia, turno, reparación, etc.) son incorporados para describir con mayor precisión los eventos conversacionales sin perder de vista los procesos discursivos que los atraviesan (Blommaert, 2005).

En tal sentido, el procesamiento de los datos fue inductivo, de manera que observamos distintas configuraciones discursivas en relación a la psiquiatría y la medicación cuya correlación con la condición laboral de los profesionales observamos en una segunda instancia.

Presentaremos los resultados del análisis en dos secciones. En la primera compararemos el empleo de formulaciones diagnósticas (Antaki et al., 2005) en relación al tratamiento psiquiátrico por parte de profesionales de planta y profesionales precarizados. En la segunda, observaremos los distintos significados ideacionales que unos y otros proyectan al emplear la pasiva perifrástica "estar medicado" u optar por construcciones activas con el verbo "medicar" o su forma nominalizada.

\subsection{Tratamiento y medicación}

Los psicoanalistas con mayor experiencia clínica, es decir, aquellos que son de planta y que ya se han socializado, de un modo u otro, con el discurso médico, consideran a la medicación psiquiátrica como parte de un tratamiento en sí mismo. Aquellos que, con menos experiencia, se oponen, en cambio, consideran que el tratamiento legítimo es el psicoanalítico y que la medicación simplemente requiere ser controlada. Analizaremos fragmentos de entrevistas conducidas por analistas con distinto grado de experiencia para observar ambos fenómenos.

\section{EXTRACTO $1^{6}$}

1. M1: Este::, a ver (.) me expusiste:: esta- este

2. pensamiento negativo de no querer

$3 . \quad$ vivir. Hiciste algo en relación a [ esto:?]

4. P1: [ No ] nunca(.)

5. No, porque siempre pensaba esto pero después dije

6. no porque me (inaudible) al psiquiatra y el

7. $\quad$ psiquiatra me dijo eres una chica muy joven, tienes

8. toda la vida por vivir=

9. M1: =Allá

10. P1: Sí, en Perú

11. M1: En Perú $(2005)$.

${ }^{6}$ Se adoptan las convenciones de trascripción propuestas por Richards y Seedhouse 
P es una mujer peruana de 23 años que se mudó a la Argentina a los veinte. Trabajó durante un año como empleada doméstica en la zona oeste del Gran Buenos Aires, lugar en el que comenzó una relación de pareja que terminó abruptamente, por un episodio que no narra. A raíz de esa "impresión fea con mi novio" describe un estado depresivo que la decide a volver a vivir con su familia en Perú. Describe discusiones familiares por una herencia y vuelve a la Argentina, seis meses antes de la consulta que estamos analizando. Durante esa estadía en Perú comienza un tratamiento psiquiátrico, que aparece referido al comienzo del fragmento.

El fragmento que analizamos se encuentra en medio de la segunda etapa de la entrevista, destinada a explicitar el motivo de la consulta. La analista presenta una formulación diagnóstica (Antaki et al., 2005) bajo la forma de una reformulación resuntiva (Arnoux, 2006) en la que selecciona información diagnósticamente relevante proporcionada por la paciente en turnos anteriores ("este pensamiento negativo de no querer vivir", 1. 2-3) y la reorienta para obtener nueva información, igualmente relevante para el diagnóstico (Bonnin, 2017). En este caso, M1 pregunta por las eventuales consecuencias prácticas de ese "pensamiento negativo", que podrían haberse traducido en un "pasaje al acto" o intento de suicidio. Aunque la formulación propuesta es lo suficientemente indefinida como para no conducir necesariamente hacia esa interpretación ("hiciste algo en relación a esto:?", 1. 3), la paciente -que cuenta con experiencia en psicoterapiada a entender que no hubo intentos de suicidio por su parte. La cadena cohesiva que vincula "este pensamiento negativo", "esto" (M, 1. 3) y "esto" $(\mathrm{P}, 1.5)$ mantiene la referencia en una implicatura conversacional: la violación de la máxima griceana de calidad permite salvaguardar la imagen positiva de la paciente y sus posibles ideaciones suicidas. $P$ introduce al psiquiatra como el actor que le dio los motivos para no intentar "esto".

Cuando $\mathrm{P}$ introduce este nuevo tópico, la psicoanalista establece, primero, los parámetros geográficos del tratamiento previo ("allá", 1. 9; "en Perú”, 1. 11) y luego busca identificar el tipo de tratamiento referido:

\section{EXTRACTO 2}

12. P1: Me ayudó bastante la verdad=

13. M1: =Y hablabas con el psiquiatra?

14. P1: Sí, [ sí] $=$

15. M1: [Bien]

16. un poco. Pero (mira) que dejé el tratamiento.

17. P1: =hablaba con el psiquiatra, él me hacía

18. $\uparrow$ preguntas

19. M1: Ahá. O sea que hiciste, aparte de control de

20. medicación, un tratamiento psicofarmacológico que 


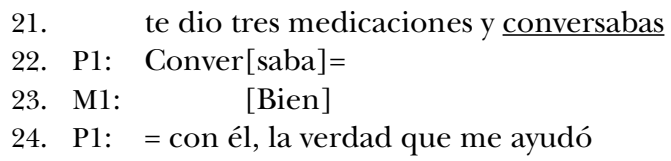

En la línea 12, P comienza a elaborar la respuesta dada dos turnos antes, de la que se desvió por la precisión geográfica requerida por M1. Ésta reorienta la interacción hacia el psiquiatra y el tratamiento que recibía la paciente, apropiándose del turno en la línea 13, sin pausa perceptible con el turno anterior. Allí introduce una secuencia Pregunta - Respuesta - Evaluación (1. 13-15) que involucra el "hablar". Reiterando las palabras de M1 en 1. 13, la paciente continúa con la elaboración interrumpida en 1. 17: "hablaba con el psiquiatra, él me hacía $\uparrow$ preguntas". Frente a la insistencia en el carácter verbal de la relación con el psiquiatra, la profesional propone una "formulación conclusiva" (upshot formulation; cfr. Antaki et al., 2005), encabezada por el conector recapitulativo "o sea", en la que distingue entre "control de medicación, un tratamiento farmacológico [en el] que te dio tres medicaciones" (1. 19-20) y “conversabas" (1. 21). Las formulaciones funcionan generalmente como pares de adyacencia con preferencia por el acuerdo (Heritage y Watson, 1979); en este caso, el acuerdo es señalado por la reiteración, "conversaba", en la línea 22, y evaluado positivamente por M1 en la línea 23.

En este pasaje queda claro, por una parte, que la acción médica atribuida al psiquiatra es definida a partir de dos componentes: "un tratamiento psicofarmacológico", por una parte, y "conversar", por otra. Este segundo elemento, a pesar de su laconismo, tiene un rol determinante en el discurso de los psicoanalistas en el hospital, puesto que es definitorio del tratamiento psicoanalítico como se practica interaccionalmente en el servicio. La misma profesional lo definía en estos términos en otra entrevista:

EXTRACTO 3

1. M1: Bueno (.) $\uparrow$ Bueno. Usted está afín de hacer un

2. tratamiento terapéutico?

3. P2: Sí

4. M1: Sabe de qué se trata?

5. P2: No, lo nunca hice.

6. M1: Bueno. (.) En realidad es esto, es como esto

7. con más tiempo de que usted pueda conversar con un

8. $\quad$ profesional $=$

9. $\mathrm{P} 2:=\mathrm{Sí}=$

10. M1: =Un terapeuta, un psicólogo o una psicóloga,

11. $\quad$ acerca de (.) bueno, las cosas que le pasan en su

$12 . \quad$ vida. 
Este ejemplo (que hemos analizado en detalle en Bonnin, 2014c) define al "tratamiento terapéutico" (es decir, la psicoterapia) como una extensión de la propia entrevista que se desarrolla: "es como esto con más tiempo de que usted pueda conversar con un profesional”. Este tipo de tratamiento, sin embargo, no tiene como agente a un psiquiatra, sino a "un psicólogo o una psicóloga”.

Observamos, entonces, que desde la perspectiva de M1 existen dos tipos de agentes profesionales cuya acción terapéutica puede ser igualmente caracterizada como "tratamiento": por una parte, el psiquiatra que provee de "el control de la medicación, un tratamiento psicofarmacológico [en el] que te dio tres medicaciones"; por otra parte, "un terapeuta, un psicólogo o una psicóloga" que da "más tiempo de que usted pueda conversar (...) acerca de (.) bueno, las cosas que le pasan en su vida".

Esta distinción categorial es sistemática, como puede observarse en el final de la entrevista a P1:

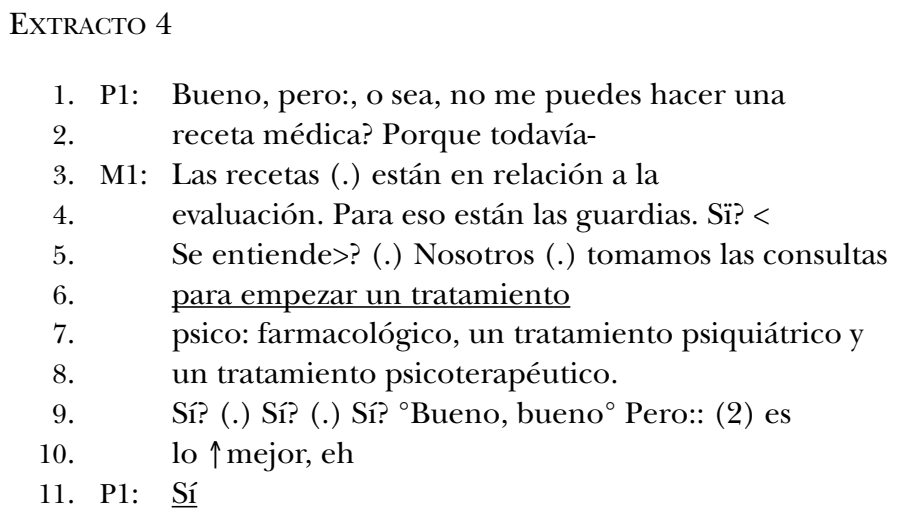

En este ejemplo, las participantes están ya transitando la última etapa de la entrevista, en la cual se ofrecen algunos consejos y recomendaciones a la espera del turno para comenzar el tratamiento. La paciente explicita, en 1. 1, la agenda latente de toda la entrevista, lo que la analista llamaría su "demanda" de medicación. Frente a esto, la profesional en 1.3 diferencia "las recetas" como una actividad que se desarrolla en "las guardias"; en oposición a esos objetos y ese agente se encuentran, nuevamente, el "tratamiento psico: farmacológico, un tratamiento psiquiátrico", por un lado, y el "tratamiento psicoterapéutico", por el otro. El agente de ambos tratamientos se define deícticamente como "nosotros", opuesto a "las guardias". Al tratarse de consultorios externos, llevan a cabo, efectivamente, tratamientos ambulatorios prolongados y no atienden casos de urgencia. Pero, más importante aún, su actividad terapéutica no consiste en hacer "rece- 
tas", sino en brindar dos tipos de tratamiento, uno de los cuales incluye medicación, pero no se agota en ella. Este tipo de profesionales, con más experiencia, puede en consecuencia adoptar la posición colectiva de la salud mental sin abandonar su propio posicionamiento como psicólogo/a.

Del otro lado, los cursistas y concurrentes tiene un alto grado de ajenidad con el discurso médico, puesto que el contacto con psiquiatras es muy esporádico. De allí que las representaciones y valoraciones acerca del tratamiento psiquiátrico sean diferentes:

\section{EXTRACTO 5}

1. D: = bue:no (.) quizás hay cosas que puede y hay

2. cosas que todavía no puede tonces, $=$

3. P2: = esta semana cumple::: veintiun años él (1) sí

4. $\quad$ es (.) sábado

5. D: sí nos había contado él (.) e:::

6. D3: (2) y: (.) acá no tenés nadie que te esté

7. $\quad$ siguiendo el control de la medicación (.)

8. una médica psiquiá:trica,

9. P: mi papá y mi mamá

10. P2: sí eso::

11. D3: sí pero quién les hace las recetas?

12. P: ustedes (2) nos lo dieron antes de ir a: $=$

13. P2: = la última sí (.) la anterior hemos ido

14. D: cla::ro pero (.) digamos no estamos haciendo un

15. tratamiento

16. P2: no no: (.) eso:

En este pasaje intervienen cuatro participantes: D y D3 son un psicólogo y una psicóloga jóvenes; el primero es un "cursista" y la segunda una "concurrente" de los consultorios externos de salud mental. A causa de la escasez de personal de planta, se encuentran a cargo de la entrevista de admisión. En el fragmento citado entrevistan a un joven boliviano de veinte años (P) que concurre acompañado por su madre (P2) y su padre, que no participa de la entrevista. Al igual que otros migrantes latinoamericanos (como P1), el paciente vivió por diversas temporadas en su país natal y en Argentina. Durante su última estancia en Bolivia, a raíz de un brote psicótico fue internado en un hospital psiquiátrico. Cuando logró el alta, se mudó a la Argentina para vivir con su familia, en un barrio del sur de la Ciudad de Buenos Aires.

En este marco, la pregunta de D3 en 1. 6-8 busca establecer si el paciente recibe atención estable con algún profesional de psiquiatría. Sin embargo, la pregunta no es por un "tratamiento psicofarmacológico" (como 
en ex. 2 y ex. 4), sino por el "control de la medicación”. Esta distancia con el empleo de medicamentos, que no aparecen como un tratamiento sino como algo externo que debe ser controlado, hace que el paciente interprete que la pregunta es por la administración de los medicamentos: "mi papá y mi mamá" (1. 9), confirmado por su madre (1. 10). Para desambiguar el malentendido, D3 describe nuevamente las tareas que serían propias de una "médica psiquiátrica", esta vez como "hace[r] las recetas" (1. 11). Frente a esta nueva pregunta, la madre responde con la referencia al sujeto "ustedes" (1. 12), designando de este modo al colectivo de profesionales de la salud mental del hospital. D interviene, entonces, para redefinir los participantes y sus acciones en la línea 14 "digamos, no estamos haciendo un tratamiento". Aquí se explicita, finalmente, la oposición entre el "control farmacológico" (a cargo de "ellos") y el "tratamiento" propiamente dicho (a cargo de "nosotros"), estableciendo deícticamente la distinción entre profesionales.

Este ejemplo puede leerse prácticamente en oposición al visto anteriormente. En ex. 5 la actividad de "una médica psiquiá:trica" (1. 8) es definida como "control de la medicación" (1. 7), es la persona que "hace las recetas" (1. 11). Para el paciente, la persona que hace las recetas es "ustedes", es decir, el colectivo de profesionales de salud mental. Para el analista, en cambio, el colectivo de los consultorios de salud mental no se encuentra comprometido en esa actividad: "[nosotros] no estamos haciendo un tratamiento" (1. 14-15).

En el ex. 4, en cambio, el colectivo de profesionales involucra, en la misma medida, "tratamiento psicofarmacológico, un tratamiento psiquiátrico" y "tratamiento psicoterapéutico", ambos incluidos en el sujeto colectivo "nosotros". "Hacer recetas", en cambio, corresponde a las guardias, y no al tratamiento de una condición crónica.

En ambos casos hubo una estrategia semejante: los pacientes, extranjeros, cruzan con frecuencia las fronteras, concurren periódicamente a la guardia de un hospital público para conseguir medicación y continúan esta suerte de tratamiento agudo de una condición crónica, con diferentes profesionales, en diferentes instituciones, con distintos fármacos, con la esperanza de aliviar síntomas inmediatos y gozar de cierta funcionalidad cotidiana. Sin advertirlo, al presentar el tratamiento psiquiátrico como mero control de la medicación, como mera factura de recetas, D y D3 admiten como clínicamente válido el control farmacológico esporádico y asistemático, puesto que no es reconocido como un "tratamiento" propiamente dicho. Los profesionales con más experiencia clínica, en cambio, proponen hacerse cargo -colectivamente- de la medicación como parte de un tratamiento más integral en salud mental. 


\subsection{Estar medicado o tomar la medicación}

¿Cómo construyen, profesionales y pacientes, la relación del sujeto con los fármacos? El término que con más frecuencia se emplea es "medicación", una nominalización del verbo "medicar" que, como toda nominalización, permite "empaquetar" información relativa al significado que el texto proyecta sobre el mundo (el "significado ideacional", Halliday, 1994: 352). De este modo, procesos, entidades y propiedades relativas a la representación del mundo que son propias del verbo y sus argumentos son "empaquetados" (Halliday y Martin, 1993: 131) en el sustantivo nominalizado y, si bien pueden reponerse con otra función sintáctica, no resultan obligatorios.

El verbo "medicar" se emplea por momentos con rasgos que lo asemejan a "recetar", por ejemplo al usarlo como verbo transitivo:

\section{EXTRACTO 6}

1. M1: (Psiquiátrico) (.) Con medicación?

2. P1: Sí:, me medicaron el (clonazepan, el sertaline y

3. el sulpiride), los tres, [de los tres]=

4. M1: [Bueno, bueno]

5. P1: =(medicamentos) me recetaron.

$\mathrm{Al}$ adoptar, en 1. 2, "clonazepam, el sertraline y el sulpiride" como complemento objeto directo, el verbo "medicar" pierde su carácter más abstracto y, en cambio, integra una construcción transitiva que sería esperable en un verbo distinto, como "recetar".

De manera semejante, la nominalización "medicación" es empleada con rasgos sintáctico-semánticos más cercanos al sustantivo "medicamento", como su carácter contable:

\section{EXTRACTO 7}

1. M1: Ahá. O sea que hiciste, aparte de control de

2. medicación, un tratamiento psicofarmacológico que

3. te dio tres medicaciones y conversabas.

4. P1: Conver[saba]=

5. M1: [Bien]

Esta tendencia a considerar contable un sustantivo que, a priori, sería un sustantivo genérico colectivo se observa en numerosos ejemplos, en los que el empleo de "medicación" alterna con el del sustantivo concreto contable "medicamento". Incluso, en el siguiente ejemplo puede obser- 
varse que la profesional realiza una autorreparación para sustituir "medicación" por "medicamento":

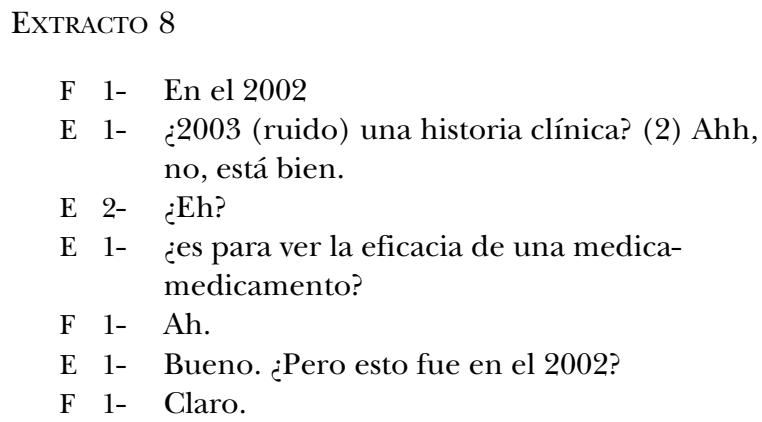

Estos ejemplos muestran que, ya sea como sustantivo o como verbo, la "medicación” admite múltiples formas alternantes que, sin embargo, son significativas. En nuestros datos hemos observado que la relación del paciente con los fármacos puede representarse de -al menos- dos maneras distintas y consistentemente recurrentes: la pasiva perifrástica "estar medicado" o la forma activa "tomar medicación/medicar". En el primer caso, el sujeto es relegado, precisamente, a una posición pasiva, en la que es definido por un estado al que lo somete la medicación o el médico:

Profesional: usted está actualmente: medicado po:r?=

Paciente: $=$ el doctor $\mathrm{X}$

Psiquiatras y psicoanalistas más experimentados no emplean la forma pasiva, que define la medicación como un estado del paciente a partir de un proceso existencial, "estar", sino que utilizan otros procesos -de conducta, materiales- que contribuyen a atribuirle una relación externa con la medicación. De esta manera, evitan poner en riesgo la imagen del paciente, que no es definido ontológicamente por la medicación, sino que simplemente la "toma" o "recibe" como algo externo a sí mismo, como parte de una acción o una conducta que desarrolla ${ }^{7}$.

Esta expresión aparece, sin embargo, con frecuencia en el discurso de los pacientes, y los profesionales con mayor experiencia clínica desarrollan diversas estrategias para evitar su empleo:

7 Esto no significa que no empleen la expresión "estar medicado", "estar re-medicado" al hablar con colegas, sino que evitan su uso al hablar con pacientes. De allí que consideremos que se trata de una estrategia conversacional referida a la negociación interaccional de la imagen, y no de un rasgo ideológico más profundo en relación a la ontología del paciente y la medicación. 
EXTRACTO 9

1. P3: Este:, estoy, eh, bastante bien medicado por el

2. doctor

3. M1: Estás contenido

4. P3: [Sí]

3. M1: $=[$ Te sentís $]$ contenido

6. P3: Sí

En el ex. 9, el paciente se describe evaluativamente (1. 1) con la forma "estar medicado", pero la profesional propone una serie de formulaciones que prácticamente reparan el turno inicial de P3. Así, en 1. 3 reinterpreta "estar bien medicado" como "estar contenido"; al lograr el acuerdo en 1. 4, superponiéndose al turno siguiente reformula el proceso existencial "estar" por el proceso mental "sentir(se)". El pasaje de "estar medicado" a "sentirse contenido" involucra una hábil maniobra verbal por parte de la analista:

a) abandona cierto discurso médico sin rechazarlo;

b) salvaguarda la imagen positiva del paciente, potencialmente amenazada por la construcción existencial "estar medicado";

c) reinterpreta clínicamente el bienestar de P3, ya no como un estado producido por los fármacos, sino como un estado de bienestar interior producto del trato con un profesional, traduciendo un proceso existencial en uno mental (cfr. Bonnin, 2017 sobre la función diagnóstica de este tipo de desplazamientos).

Profesionales con poca experiencia y una socialización clínica insuficiente, en cambio, suelen emplear la pasiva "estar medicado/a" al describir la situación farmacológica del paciente:

\section{EXTRACTO 10}

1. E- Ya habló de un espacio para hablar.

2. ¿Qué piensa de eso? (en el cuerpo de cosas) que le

3. pasan, sus angustias, esos miedos que tiene a la

4. noche, estos síntomas que aparecieron de nuevo.

5. Porque con algo lo t- se pueden relacionar me parece

6. (1) ¿Usted está medicada actualmente?

7. F 2- No. No(1)

8. E- ¿No? O sea, usted estuvo medicada en el 2002 y::,

9. durante un tiempo y después dejó la medicación.

10. F 2- Claro. No. Desde. Yo me controlaba del

11. psiquiatra la medicación

En este ejemplo, una mujer de sesenta años, que vive en la zona sur del conurbano bonaerense, se presenta a raíz de una serie de episodios 
que identifica como "ataques de pánico". F ya había recibido tratamiento psiquiátrico diez años atrás, cuando participó como sujeto experimental en el testeo de una droga para la ansiedad en un hospital de Capital Federal. Durante la entrevista, la paciente muestra una gran familiaridad con la voz médica, la cual adopta en diversos pasajes al negociar la asimetría de roles con la psicoanalista que conduce la entrevista (Bonnin, 2014a). La profesional, por su parte, es una joven concurrente que se encuentra por primera vez a cargo de una entrevista de admisión, habiendo participado como asistente en otras durante ese mismo año. Quizás por ese motivo, la agenda que construye no responde a la actividad rutinaria de la entrevista, sino que incluye preguntas esperables en la primera sección ("¿está medicada actualmente?", 1. 6) en la segunda fase de la entrevista, destinada a los motivos de consulta. La expresión "estar medicada" es empleada dos veces por E, tanto para referirse a la situación presente (1. 6) como para el pasado (1. 8). Este empleo es consistente con el uso que hemos observado en los pacientes (cfr. ex. 9), pero no con los profesionales de mayor experiencia. De hecho, la paciente adopta en 1. 10-11 la expresión "control de la medicación” que, como vimos en la sección anterior, se corresponde también con los profesionales de menor experiencia. De esta manera, la construcción pasiva "estar medicada/o" coincide con el rol secundario atribuido a la psiquiatría como mero "control de la medicación".

\section{Conclusiones}

Los estudios sobre socialización profesional en el área de salud muestran la importancia que tiene en el desarrollo de saberes sobre la institución y la interdisciplina, sobre la interacción (Peräkylä et al., 2008) y la construcción de voz e identidad profesional (Bonnin, 2014c). Este tipo de socialización se encuentra subordinada al tiempo insumido en el trabajo, y el tiempo destinado depende del tipo de relación laboral. ¿Qué sucede cuando el trabajo se produce en condiciones de precariedad, en una relación no salarial que condiciona las posibilidades de participación efectiva en las diversas instancias y prácticas de socialización profesional? En este trabajo hemos observado que esa precariedad produce una socialización insuficiente en la adquisición de saberes y representaciones acerca del tratamiento psiquiátrico, la cual se manifiesta en la propia interacción con los pacientes y puede, en consecuencia, incidir en la práctica clínica que efectivamente se lleva a cabo.

El trabajo presentado muestra diferencias sistemáticas en las representaciones sobre el tratamiento psiquiátrico y la medicación producidas por profesionales de planta y precarizados en la interacción con pacien- 
tes en entrevistas de admisión en salud mental. Aquellos que cuentan con más experiencia designan la actividad del psiquiatra como un tratamiento (psico)farmacológico y evitan emplear formas pasivas para referirse al consumo de la medicación por parte del paciente. Los que tienen menos experiencia, en cambio, denominan control farmacológico/de la medicación a la primera, y emplean la forma pasiva, estar medicado/a, para referirse al consumo de medicación por parte del paciente. La pasiva perifrástica "estar medicado" produce el efecto opuesto al deseado por los jóvenes profesionales: aunque se proponen desmedicalizar, en realidad naturalizan el hecho y dejan al paciente en un lugar de pasividad.

Ambas representaciones y, en consecuencia, modos de interacción impactan en la percepción que el paciente desarrolla del tratamiento en salud mental: si el "control de la medicación" no es un tratamiento propiamente dicho, puede seguir sosteniéndose esporádicamente por medio de las guardias médicas. De allí que "hacer recetas" sea una actividad en disputa, que los psicólogos con más experiencia intentan sustituir por un "tratamiento farmacológico" propiamente dicho. 


\section{BIBLIOGRAFÍA}

Antaki, C., Barnes, R. y Leudar, I. (2005): "Diagnostic formulations in psychotherapy”, Discourse Studies, 7 (6), págs. 627-647.

Antman, J. (2007): "Los Concurrentes de Salud Mental de la Ciudad de Buenos Aires". Disponible en: <http://www.elpsitio.com.ar/Noticias/NoticiaMuestra. asp? Id $=1703>$.

Antman, J. (2003): "La Concurrencia de Salud Mental en la Ciudad de Buenos Aires". Disponible en: <https://www.topia.com.ar/articulos/concurrenciasalud-mental-ciudad-buenos-aires $>$.

Arnoux, E. B. N. (2006): Análisis del Discurso. Modos de abordar materiales de archivo, Buenos Aires, Santiago Arcos.

BonnIN, J. E. (2013): "The public, the private and the intimate in doctor-patient communication: Admission interviews at an outpatient mental healthcare service", Discourse Studies, 15 (6), págs. 687-711.

Bonnin, J. E. (2014a): "To speak with the other's voice: reducing asymmetry and social distance in professional-client communication", Journal of Multicultural Discourses, 9 (2), págs. 149-171.

Bonnin, J. E. (2014b): "Expanded answers to bureaucratic questions: Negotiating access to public healthcare", Journal of Sociolinguistics, 18 (5), págs. 685-707.

Bonnin, J. E. (2014c): "Treating without diagnosis: Psychoanalysis in medical settings in Argentina”, Communication and Medicine, 11 (1), págs. 15-26.

Bonnin, J. E. (2017): "Formulations in Psychotherapy: Admission Interviews and the Conversational Construction of Diagnosis", Qualitative Health Research, 27, págs. 1-9.

Blommaert, J. (2005): Discourse: a critical introduction, Cambridge, Cambridge University Press.

Bolívar, A. (2010): "A change in focus: from texts in contexts to people in events", Journal of Multicultural Discourses, 5 (3), págs. 213-225.

Candlin, C. y Candlin, S. (2003): "Health care communication: A problematic site for applied linguistics research", Annual Review of Applied linguistics, 23, págs. 134-154

Candlin, S. y Candlin, C. (2007): "Nursing through Time and Space: Some Challenges to the Construct of Community of Practice", en R. Iedema (ed.), The Discourse of Hospital Communication. Tracing Complexities in Contemporary Health Care Organizations, Basingstoke, Palgrave Macmillan, págs 244-267.

Cicourel, A. (1999): "The interaction of cognitive and cultural models in health care delivery", en S. Sarangi y C. Roberts (eds.), Talk, Work and Institutional Order: Discourse in Medical, Mediation and Management Settings, Berlin-New York, Mouton de Gruyter, págs. 183-224.

ClaRK, P. G. (1997): "Values in health care professional socialization: implications for geriatric education in interdisciplinary teamwork", Gerontologist, 37 (4), págs. 441-451. 
Clouder, L. (2003): "Becoming professional: exploring the complexities of professional socialization in health and social care", Learning in Health and Social Care, 2 (4), págs. 213-222.

CONWAY, M. E. (1983): "Socialization and roles in nursing", Annual Review of Nursing Research, 1, págs. 183-208.

Cordella, M. (2004): The Dynamic Consultation. A discourse analytical study of doctorpatient communication, Amsterdam/Philadelphia, John Bejamins.

Crawford, P., Brown, B. y Mullany, L. (2005): "Clinical governmentality: A critical linguistic perspective on clinical governance in health care organizations", Journal of Applied Linguistics, 2 (3), págs. 273-298.

Fochsen, G., Deshpande, K. y Thorson, A. (2006): "Power Imbalance and Consumerism in the Doctor-Patient Relationship: Health Care Providers' Experiences of Patient Encounters in a Rural District in India”, Qualitative Health Research, 16 (9), págs. 1236-1251.

Fox, F. E., Rodham, K. J., Harris, M. F., Taylor, G. J., Sutton, H., Scotton, J. y Robinson, B. (2009): "Experiencing 'The Other Side': A Study of Empathy and Empowerment in General Practitioners Who Have Been Patients", Qualitative Health Research, 19 (11), págs. 1580-1588.

HallidaY, M. A. K. (1994): An introduction to functional grammar, Londres, Arnold.

HALlidAY, M. A. K. y MARTIN, J. R. (1993): Writing science: literacy and discursive power, Pittsburgh, University of Pittsburgh Press.

Heritage, J. y Clayman, S. (2010): Talk in Action. Interactions, Identities and Institutions, Oxford, Wiley-Blackwell.

Heritage, J. y MaYnard, D. W. (eds.) (2006): Communication in Medical Care. Interaction between primary healthcare physicians and patients, Cambridge, Cambridge University Press.

Heritage, J. y Watson, R. (1979): "Formulations as Conversational Objects", en G. Psathas (ed.), Everyday Language, New York, Irvington Press, págs. 123-162.

IEDEMA, R. (2005a): "The tension between professional and institutional discourse: An applied linguistic analysis of hospital communication", Journal of Applied Linguistics, 2 (3), págs. 243-252.

IEDEMA, R. (2005b): "Critical incident reporting and the discursive reconfiguration of feeling and positioning”, Journal of Applied Linguistics, 2 (3), págs. 325-349.

Iedema, R. (ed.) (2007): The Discourse of Hospital Communication. Tracing Complexities in Contemporary Health Care Organizations, Basingstoke, Palgrave Macmillan.

LAKoff, A. (2006): Pharmaceutical reason: knowledge and value in global psychiatry, Cambridge, Cambridge University Press.

MAY, C. (2007): "The Clinical Encounter and the Problem of Context", Sociology, 41 (1), págs. 29-45.

Moyer, M. G. (2011): "What multilingualism? Agency and unintended consequences of multilingual practices in a Barcelona health clinic", Journal of Pragmatics, 43 (5), págs. 1209-1221.

Moyer, M. G. y Codó, E. (2002): "Language and dynamics of identity among immigrants. Challenging practices of social categorization in an institutional setting”, ponencia presentada en II Simposio Internacional Bilingüismo, Vigo, España. Disponible en: <http://webs.uvigo.es/ssl/actas2002/08/03.\%20Me lissa\%20G.\%20Moyer.pdf $>$. 
Novick, M. y Galin, P. (2003): "Flexibilización del mercado de trabajo y precarización del empleo. El caso del sector salud”, en AA.VV., Observatorio de Recursos Humanos en Salud en Argentina. Información estratégica para la toma de decisiones, Buenos Aires, Organización Panamericana de la Salud, págs. 11-90.

Organización PANAmERICANa de la SALUd (2012): Estudio comparativo de las condiciones de trabajo y salud de los trabajadores de la salud en: Argentina, Brasil, Costa Rica y Perú, Washington, Organización Panamericana de la Salud.

Pardo, M. L. y Buscaglia, V. (2008): "Pobreza y salud mental desde el Análisis Crítico del Discurso. El aislamiento social y el deterioro comunicativo y cognitivo", Discurso y Sociedad, 2 (2), págs. 357-393.

PARdo, M. L. y LERNER, B. (2001): "El discurso psicótico: una visión multidisciplinaria desde la Lingüística y la Psiquiatría”, Signos, 34 (49-50), págs. 139-148.

PARsons, T. (1975): "The Sick Role and the Role of the Physician Reconsidered", The Milbank Memorial Fund Quarterly. Health and Society, 53 (3), págs. 257-278.

Peck, B. M. y Denney, M. (2012): "Disparities in the Conduct of the Medical Encounter: The Effects of Physician and Patient Race and Gender", Sage Open, 2, págs. 1-14.

Peräkylä, A., Antaki, C., Vehilläinen, S. y Leudar, I. (2008): "Analysing Psychotherapy in Practice”, en A. Peräkylä, C. Antaki, S. Vehilläinen e I. Leudar (eds.), Conversation Analysis and Psychotherapy, Cambridge, Cambridge University Press, págs. 5-25.

PERÄKYLÄ, A. (2008): "Psychoanalysis and Conversation Analysis: Interpretation, Affect and Intersubjectivity”, en A. Peräkylä, C. Antaki, S. Vehilläinen e I. Leudar (eds.), Conversation Analysis and Psychotherapy, Cambridge, Cambridge University Press, págs. 100-119.

Richards, K. y SEEDHOUSE, P. (2005): Applying conversation analysis, HoundmillsBasingstoke-New York, Palgrave-Macmillan.

SARANGI, S. (2004): "Towards a communicative mentality in medical and healthcare practice", Communication \& Medicine, 1 (1), págs. 1-11.

SARANGI, S. (2011): "Role hybridity in professional practice", en S. Sarangi, V. Polese y G. Caliendo (eds.), Genre(s) on the Move: Hybridisation and Discourse Change in Specialised Communication, Napoli, Edizioni Scientifiche Italiane, págs. 271-296.

SARANGI, S. y RoberTs, C. (1999): "Introduction: Discursive hybridity in medical work", en S. Sarangi y C. Roberts (eds.), Talk, Work and Institutional Order. Discourse in Medical, Medication and Management Settings, Berlin-New York, Mouton de Gruyter, págs. 1-60.

Stivers, T. y Heritage, J. (2001): "Breaking the sequential mould: Answering 'more than the question' during comprehensive history taking”, Text, 21 (1-2), págs. 151-185.

WodAK, R. (2006): “Medical Discourse: Doctor-Patient Communication”, en K. Brown (ed.), Encyclopedia of Language \& Linguistics, VII, Oxford, Elsevier, págs. 681-287. 